OPEN ACCESS

Edited by:

Laishuan Wang,

Fudan University, China

Reviewed by:

Johannes Boltze,

University of Warwick,

United Kingdom

Martin Mueller,

University Hospital of Bern,

Switzerland

*Correspondence:

Wise Young

wisey@mac.com

Wai Sang Poon

wpoon@surgery.cuhk.edu.hk

Specialty section:

This article was submitted to

Cellular Neuropathology,

a section of the journal

Frontiers in Cellular Neuroscience

Received: 27 November 2021

Accepted: 13 January 2022

Published: 02 March 2022

Citation:

Lyu H, Sun DM, Ng CP,

Cheng WS, Chen JF, He YZ, Lam SY, Zheng ZY, Huang GD, Wang CC,

Young W and Poon WS (2022)

Umbilical Cord Blood Mononuclear

Cell Treatment for Neonatal Rats With

Hypoxic Ischemia.

Front. Cell. Neurosci. 16:823320

doi: 10.3389/fncel.2022.823320

\section{Umbilical Cord Blood Mononuclear Cell Treatment for Neonatal Rats With Hypoxic Ischemia}

\author{
Hao Lyu ${ }^{1,2}$, Dong Ming Sun 3 , Chi Ping Ng ${ }^{1}$, Wendy S. Cheng', Jun Fan Chen ${ }^{1}$, \\ Yu Zhong He' ${ }^{1}$, Sin Yu Lam ${ }^{1}$, Zhi Yuan Zheng ${ }^{1,5}$, Guo Dong Huang ${ }^{2}$, Chi Chiu Wang ${ }^{6}$, \\ Wise Young ${ }^{3 *}$ and Wai Sang Poon ${ }^{*}$

\begin{abstract}
'Division of Neurosurgery, Department of Surgery, Faculty of Medicine, The Chinese University of Hong Kong, Shatin, Hong Kong SAR, China, ${ }^{2}$ Shenzhen Key Laboratory of Neurosurgery, Department of Neurosurgery, The Shenzhen Second People's Hospital, First Affiliated Hospital of Shenzhen University, Shenzhen, China, ${ }^{3}$ W. M. Keck Center for Collaborative Neuroscience, Rutgers, The State University of New Jersey, Piscataway, NJ, United States, ${ }^{4}$ Mononuclear Therapeutics Limited, Hong Kong, Hong Kong SAR, China, ${ }^{5}$ Department of Neurosurgery, Hainan Hospital of People's Liberation Army General Hospital, Sanya, China, ${ }^{6}$ Department of Obstetrics and Gynecology, Li Ka Shing Institute of Health Sciences, School of Biomedical Sciences, Chinese University of Hong Kong-Sichuan University Joint Laboratory in Reproductive
\end{abstract} \\ Medicine, Shatin, Hong Kong SAR, China
}

Background: Hypoxic-ischemic encephalopathy (HIE) occurs when an infant's brain has not received adequate oxygen and blood supply, resulting in ischemic and hypoxic damage. Currently, supportive care and hypothermia therapy have been the standard treatment for HIE. However, there are still over $20 \%$ of treated infants died and $19-30 \%$ survived with significant disability. HIE animal model was first established by Rice et al., involving the ligation of one common carotid artery followed by hypoxia. In this study, we investigated human umbilical cord blood (HUCB) and its two components mononuclear cell (MNC) and red cell fraction (RCF) in both short and long term study using a modified HIE rat model.

Methods: In this modified HIE model, both common carotid arteries were occluded, breathing $8 \%$ oxygen in a hypoxic chamber for $60-\mathrm{min}$, followed by the release of the common carotid arteries ligature, mimicking reperfusion injury. For cell therapeutic study, cells were intravenously injected to HIE rat pups, and both behavioral and histological changes were assessed at selected time points.

Result: Statistically significant behavioral improvements were demonstrated on Day 7 and 1 month between saline treated HIE rats and UCB/MNC treated rats. However, at 3 months, the therapeutic improvements were only showed between saline treated HIE animals and MNC treated HIE rats. For histological analysis 1 month after cell injection, the number of functional neurons were statistically increased between saline treated $\mathrm{HIE}$ and $\mathrm{UCB} / \mathrm{MNC} / \mathrm{RCF}$ treated HIE rats. At 3 months, the significant increase in functional neurons was only present in MNC treated HIE rats. 
Conclusion: We have used a bilateral temporary occlusion of 60 min, a moderately brain damaged model, for cell therapeutic studies. HUCB mononuclear cell (MNC) therapy showed benefits in neonatal HIE rats in both short and long term behavioral and histological assessments.

Keywords: hypoxic ischemia encephalopathy (HIE), animal model, cell therapeutic, umbilical cord blood (UCB), mononucleal cells

\section{INTRODUCTION}

Hypoxic-ischemic encephalopathy (HIE) occurs when the infant brain is deprived of oxygen due to limited blood flow. It is a major cause of neurological deficits in new-born babies. The incidence of HIE is approximately 5 per 1000 live births worldwide (Kurinczuk et al., 2010). It is characterized by edema, secondary cellular energy failure and excitotoxicity with a major mitochondrial dysfunction, which triggers cell death and clinical complications of moderate to severe damage in the brains of the new-born (Gunn et al., 1997; Bennet et al., 2012). Of the neonates who survive the HIE, 20\% develop cerebral palsy (CP) (Lehnardt et al., 2003; Gluckman and Rocha, 2004; Gluckman et al., 2005; Eklind et al., 2005; Shankaran et al., 2005; Jacobs et al., 2011).

Immediate supportive care is of paramount importance in the resuscitation of these HIE babies by restoring sufficient cerebral blood flow (CBF), maintaining adequate oxygen and glucose supply to the brain, and finally preventing further brain injury (Douglas-Escobar and Weiss, 2015). Hypothermia therapy has been used for term infants with HIE. This treatment involves decreasing the neonate's body temperature to $33-35^{\circ} \mathrm{C}$, and has been proven to reduce the risks of neurodevelopmental deficits and death in a long-term study of neonates with moderate to severe HIE (Shankaran et al., 2005; Gunn et al., 2008; Azzopardi et al., 2009).

Despite receiving the best medical care, including hypothermia therapy, approximately $20 \%$ of infants with HIE die or survive with moderate to severe neurological disabilities, such as CP (Shankaran et al., 2005; Graham et al., 2016). Hypothermia therapy is effective only when administered within $6 \mathrm{~h}$ after birth (Shankaran et al., 2005; Higgins et al., 2011). Therefore, novel therapeutic approaches, especially those with a long therapeutic window, are warranted for infants with HIE.

Cell therapy has received increasing attention in the past two decades, not only for its regenerative ability but also for its therapeutic window (Jantzie et al., 2018). Human umbilical cord blood (HUCB) consists of a large amount of monocytes (40\%), lymphocytes (40\%), neutrophils (10\%), and the remaining $10 \%$, a mixture of endothelial progenitor cells, multipotent stem cells, as well as the mesenchymal stem cells (MSCs), similar to the bone marrow cell population (Jaing, 2014). In the literature, these MNCs in HUCB have been reported to consist of 3-10\% immature progenitor cells (Jaing, 2014; Zhu et al., 2016). Moreover, it can be readily collected and stored

Abbreviations: HIE, hypoxic-ischemic encephalopathy; HUCB, human umbilical cord blood; MNC, mononuclear cells; RCF, red cell fraction; CP, cerebral palsy; CBF, cerebral blood flow; MSC, mesenchymal stem cells; CCA, common carotid arteries. for future therapy and research. The immune rejection risk is low in HUCB transplantation as its HLA antigen levels remain low in comparison with cells harvested from adults, such as bone marrow derived MSCs (Penny et al., 2019). HUCB was first used in animal models of HIE by Meier and her colleagues. Ten million HUCB MNCs in $0.5 \mathrm{ml}$ was delivered intraperitoneally into the postnatal day 7 rat pups $24 \mathrm{~h}$ after HIE. The Meier study has demonstrated the presence of MNCs in the affected cerebral hemispheres of the treated HIE pups, without neuronal or astrocytic differentiation (Meier et al., 2006). Another study found that intravenous injection of a small dose of HUCB MNCs $(15,000) 7$ days after HIE, assisted by blood brain barrier manipulation with intravenous mannitol, improved motor coordination of these MNCs-treated rat pups (Yasuhara et al., 2010). A clinical research group at Duke University tested the feasibility and safety of transplanting autologous HUCB in neonates with HIE. The efficacy of the cell therapy was assessed based on neurological development scores until the recruited infants were 1-year-old (Pimentel-Coelho and Mendez-Otero, 2010; Cotten et al., 2014).

However, several aspects of HUCB as a treatment for HIE remain unclear, including the mechanisms of its therapeutic effects, the cell types that contribute the most to this effect and the optimal cell transplantation route, number of cells and timing of transplantation. Therefore, in this study, we investigated the effects of transplantation with total HUCB and its two components, mononuclear cells (MNCs) and the red cell fraction (RCF), over the short term (7 days after cell transplantation) and long term ( 1 and 3 months after cell transplantation) in a modified HIE rat model (Lyu et al., 2021).

\section{MATERIALS AND METHODS}

\section{Human Umbilical Cord Blood, Mononuclear Cell, and Red Cell Fraction Preparation}

The collection of Umbilical Cord Blood (UCB) was approved by the Joint Chinese University of Hong Kong-New Territories East Cluster Clinical Research Ethics Committee (CREC Reference Number 2016.250). Blood was collected from the umbilical cord and connected placenta from consented mothers in the Department of Obstetrics and Gynecology, The Prince of Wales Hospital, The Chinese University of Hong Kong. The mother has provided signed consent for the use of these materials. The blood was collected in bags containing anticoagulant and will be stored in liquid nitrogen if no immediate clinical or research 
requests were made. $\mathrm{HUCB}, \mathrm{MNC}$, and the RCF were prepared by density-gradient centrifugation based on laboratory protocols. The cells were re-suspended in $0.9 \%$ sodium chloride, and their cell numbers were determined. The cell viabilities were confirmed to be higher than $90 \%$. The qualified collections of HUCB were processed by our collaborator Mononuclear Therapeutics Ltd (MT, Hong Kong SAR, China) using the latest SynGeneX ${ }^{\circledR}-1000$ system and CryoPRO-5 Processing and Storage Bag (SynGen Inc, San Carlo, CA, United States).

\section{The Modified Hypoxic-Ischemic Encephalopathy Animal Model}

Hypoxic-ischemic encephalopathy animal model was first established by Rice et al. (1981), and has been used extensively to explore the mechanisms of brain damage resulting from HIE and to test the effectiveness of potential therapeutic interventions. The HIE model involves the ligation of one common carotid artery followed by hypoxia ( $8 \%$ oxygen), however, this model did not simulate the real situation of human HIE. In this study, we used a modified bilateral temporary occlusion HIE animal model as we described recently, which better mimics the human situation of a difficult labor (Lyu et al., 2021). All animal experimentation protocols followed the guidelines for the care and use of animals and were approved by the ethics committee of the Chinese University of Hong Kong. The modified bilateral HIE model was used to induce the brain damage from hypoxia and ischemia in the 7-day-old neonatal rats. All animals were housed under a 12-h light/12-h dark cycle with free access to food and water. The rats were fully anesthetized with isoflurane (3-4\% for induction and $1-2 \%$ for maintenance), and a small incision was made in the middle of the neck using an \#11 scalpel. Using blunt dissection, the connective tissue and muscles were separated to clearly visualize blood vessels under an operating microscope. To establish the bilateral HIE model, both common carotid arteries (CCA) were temporally ligated with 6-0 silk suture. After the incision was closed with a suture, the pups were placed in a hypoxia chamber containing $8 \%$ oxygen balanced with $92 \%$ nitrogen for $60 \mathrm{~min}$. A heating pad was used to maintain the pups' body temperature in the chamber. At the end of hypoxia exposure, the pups were removed from the chamber and anesthetized again with isoflurane. Their incisions were opened, and the ligatures on both CCAs were released. The incisions were then closed with 6-0 sutures, and the pups were allowed to wake up in a recovery box. Buprenorphine (Buprenex) $(0.05 \mathrm{mg} / \mathrm{kg})$ was administered to the pups for $6 \mathrm{~h}$ for postoperative analgesia.

\section{Experimental Grouping}

The animals were assigned randomly to six experimental groups: Normal control (no brain injury), HIE only, HIE with sham injection (with $0.9 \%$ sodium chloride), and HIE with cell therapy (HUCB, MNC or the RCF).

Animals in the HIE with sham injection group were intravenously injected with $200 \mu \mathrm{L}$ of $0.9 \%$ sodium chloride $24 \mathrm{~h}$ after HIE modeling (i.e., bilateral CCA occlusion followed by hypoxia). In the cell therapy group, the rat pups were intravenously injected with $1 \times 10^{7}$ of UCB, MNC or RCF in the selected volume of $200 \mu \mathrm{L}$ or $0.2 \mathrm{ml}, 24 \mathrm{~h}$ after HIE modeling.

\section{Cell Transplantation}

For cell transplantation, the rat pups in the cell therapy group were fully anesthetized with isoflurane (3-4\% for induction and $1-2 \%$ for maintenance), and a small incision was made in the left side of the neck using an \#11 scalpel. Using blunt dissection, the connective tissue and muscles were separated to clearly visualized the left external jugular vein under a microscope. Using a 30G insulin syringe, $1 \times 10^{7}$ cells $(200 \mu \mathrm{L}$ or $0.2 \mathrm{ml})$ were slowly injected in the direction of the heart over a period of $10 \mathrm{~min}$ to avoid complications due to fluid overload. Subsequently, the syringe was withdrawn, compression was applied to the wound for $5 \mathrm{~min}$ to stop bleeding. The incision was closed with one suture.

\section{Real-Time PCR}

Human Alu Yb8 was detected 2 days after cell transplantation to determine the migration of HUCB and MNC in the pups' brains. The rat pups were anesthetized with ketamine $(75 \mathrm{mg} / \mathrm{kg})$ and xylazine $(5 \mathrm{mg} / \mathrm{kg})$ and subjected to transcardiac perfusion with $0.9 \%$ sodium chloride followed by RNA later (Invitrogen $^{\mathrm{TM}}$ RNAlater $^{\mathrm{TM}}$ Stabilization Solution, AM7021). Total DNA was extracted from the brain, heart, liver, lungs, stomach, spleen and kidneys using a DNA extraction kit (Qiagen 69506) according to the manufacturer's instructions. The standard curve was prepared. The primer set for the human Alu Yb8 sequence (sense 5'-CGAGGCGGGTGGATCATGAGGT-3'; antisense $5^{\prime}$-TCTGTCGCCCAGGCCGGACT-3') was provided by our collaborator at Rutgers University (United States). Realtime PCR was performed with $300 \mathrm{ng}$ of DNA and PowerUp SYBR Green Master Mix (Thermo Fisher, New York, NY, United States, A25741) using the QuantStudio ${ }^{\mathrm{TM}} 12 \mathrm{~K}$ Flex System under the following conditions: $50^{\circ} \mathrm{C}$ for $2 \mathrm{~min}$ and $95^{\circ} \mathrm{C}$ for $10 \mathrm{~min}$, followed by 40 cycles of $95^{\circ} \mathrm{C}$ for $15 \mathrm{~s}, 62^{\circ} \mathrm{C}$ for $15 \mathrm{~s}$ and $72^{\circ} \mathrm{C}$ for $30 \mathrm{~s}$. The PCR data were analyzed using the Thermo Fisher online system ${ }^{1}$.

\section{Behavioral Assessments}

The colleagues who conducted the behavioral tests were all blinded to the animal experimentation grouping.

\section{Short-Term Assessments Negative Geotaxis}

To investigate the short-term effects of cell therapy on HIE, the negative geotaxis of the rat pups in all groups was tested on day 7 after cell transplantation. Briefly, the pups were placed on an inclined board $\left(40^{\circ}\right)$ with their head in the downward position. The time taken by the pups to turn $180^{\circ}$ was recorded up to a maximum cut-off time of 60 s; i.e., the time to rotate for the pups that failed to rotate was recorded as $60 \mathrm{~s}$.

\footnotetext{
${ }^{1}$ https://apps.thermofisher.com/apps/spa/\#/dashboard
} 


\section{Long-Term Assessments}

To investigate the long-term effects of cell therapy, the pups in all groups were subjected to the beam balance test to assess motor function assessment and the Morris water maze test to assess spatial learning memory at 1 and 3 months after cell transplantation.

\section{Beam Balance}

For the beam balance test, a $100-\mathrm{cm}$-long, 1.75-cm-wide beam was set $90 \mathrm{~cm}$ above the floor. The test was conducted in an empty cage, and bedding was placed under the beam to protect the pups from injury if they fell. On the testing day, the rat pups were placed on the beam for $1 \mathrm{~min}$, and the duration on the beam and balance score of each pup were recorded. Scoring was determined according to the following scale: (1) a stable balance posture; (2) a shaky posture indicated by grasping of the beam; (3) a balancing attempt by hanging on the beam; (4) falling off the beam after $10 \mathrm{~s}$; (5) balancing attempt by hanging on the beam, followed by falling off the beam in $10 \mathrm{~s}$; and (6) falling off the beam without hanging on. Each rat pup was tested three times, with a resting interval of a 5 min between each attempt.

\section{Morris Water Maze}

As the hippocampus was affected first after HIE, we assessed spatial-learning memory which correlated to hippocampus function. For this test, a $200-\mathrm{cm}$-diameter maze and a $10-\mathrm{cm}$ diameter escape platform were used, with four visual direction symbols placed as cues on the four tank walls. The water was maintained at room temperature. A camera connected to a computer was secured on the tank to record the pups' swimming routes. The water tank was divided into four quadrants labeled as northeast (NE), southeast (SE), southwest (SW), and northwest (NW), and the escape platform was placed in the NW quadrant. Each rat pup was tested in four trials; the pup was placed facing the tank wall in a different quadrant in each trial, starting clockwise from NE to NW. The time taken by the pup to find the escape island in each trial (from each starting quadrant) was recorded. The cut-off time to reach the island was set at $120 \mathrm{~s}$, and a 15-s interval was allowed between each trial (Vorhees and Williams, 2006). For this trial, the escape island was removed, and the animal was placed facing the tank wall at a new start position, viz. in the quadrant (SE quadrant) opposite to that in which the island was originally located (NW quadrant). The cut-off time to complete the trial was set at $60 \mathrm{~s}$. A new start position in the probe trial ensures that the testing animal's spatial preference is a reflection of the memory of the goal location, rather than a preference for a specific swimming path. The cumulative distance (distance between the pup and the center of the island at every $200 \mathrm{~ms}$ ) and island crossing (number of times the pup entered the island location) were recorded to evaluate the pups' reference memory.

\section{Immunohistochemistry}

After behavioral assessments on day 7 and at 1 and 3 months, the rat pups were anesthetized by ketamine $(75 \mathrm{mg} / \mathrm{kg})$ and xylazine $(5 \mathrm{mg} / \mathrm{kg}$ ) and then subjected to transcardiac perfusion with $0.9 \%$ sodium chloride followed by $4 \%$ paraformaldehyde (PFA).
The brains were removed, stored in 4\% PFA for $24 \mathrm{~h}$ and then dehydrated in an alcohol gradient (50, 70, 90, and 100\%). The dehydrated brains were embedded in paraffin, and $5-\mu \mathrm{m}$-thick sections were cut.

For the immunohistochemistry analysis, paraffin-embedded sections were stained with anti-NeuN primary antibody (Millipore, Burlington, MA, United States, MAB377, 1:200 dilution) at $4^{\circ} \mathrm{C}$ overnight then incubated with a secondary antibody (Abcam, Cambridge, United Kingdom, ab97023, Goat Anti-Mouse IgG, HRP, 1:500) for $2 \mathrm{~h}$ at room temperature. Subsequently, the sections were washed with PBS two times and then stained with 3,3'-diaminobenzidine for immunohistochemical analysis. Positive cell signals were recorded using an imaging microscope (Nikon Fi-3). For the quantitative analysis of NeuN-positive cells, six $0.5-\mathrm{mm}^{2}$ squares in motor cortex were selected (three continuous slides per rat) and NeuN-positive cells were counted using Nikon NIS Element BR software.

\section{Statistical Analysis}

GraphPad Prism 8.3.0 software was used for statistical analysis and graphics production. A one-way ANOVA with Tukey's multiple comparison test was performed to assess the results of the behavioral tests and immunohistochemistry analysis. A $p$-value of $<0.05$ was considered significant to reject the null hypothesis. Normal distribution was checked by Kolmogorov-Smirnov test, a $p$-value of $>0.1$ was considered pass the normality test.

\section{RESULTS}

\section{Animal Sex, Number, Mortality and Grouping}

The neonatal rats were un-sexed chosen, of which $42 \%$ were female. The mortality of HIE was $30 \%$ which is consist with our previous study (Lyu et al., 2021), and similar with the human situation (Shankaran et al., 2005). A total of 230 animals survived the HIE. Each neonatal rat was assigned randomly to six experimental groups: Normal control ( $n=12$ per group), HIE only ( $n=12$ per group), HIE with sham injection (transplanted with $0.9 \%$ sodium chloride, $n=12$ per group), and HIE with cell therapy (HUCB, MNC or the RCF, $n=12$ per group). The mortality of transplantation was $10 \%$. Animals with too much bleeding during HIE surgery, intravenous injection, as well as blood clot found on section slides were excluded.

\section{Expression of Human Alu Yb8}

Human UCB-derived MNC $\left(1 \times 10^{7}\right)$ were injected intravenously into the rat pups $24 \mathrm{~h}$ after HIE modeling. The Alu Yb8 marker specific to these cells was detected in several organs, including the brains of the pups $(n=3)$. A standard curve was generated to estimate the number of cells based on the cycles that reach to plateau phase (Figure 1A). The results showed that human Alu Yb8 was detectable in all of the selected organs 2 days after intravenous cell injection, indicating that 
A

\section{Standard Curve}

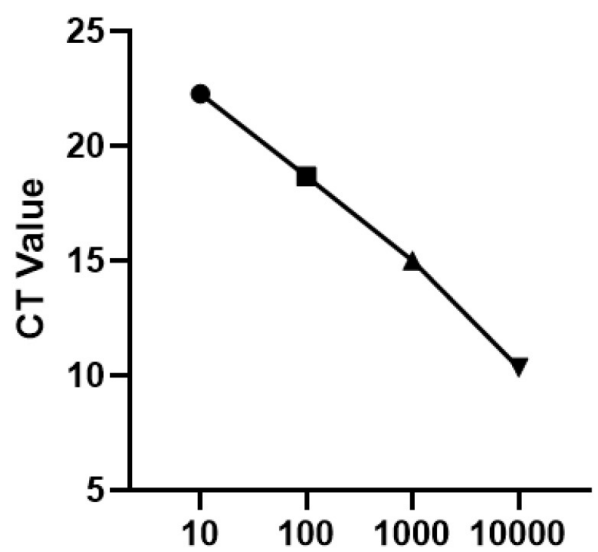

B

\section{Jugular Vein Injection}

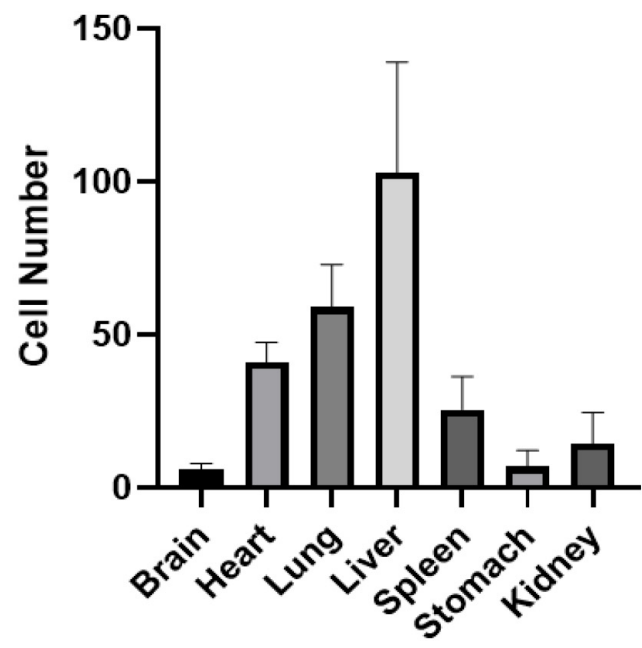

FIGURE 1 | (A) Standard curve and (B) expression of human Alu Yb8 in selected organs based on real-time PCR results ( $n=3$ rat pups). The liver showed the highest number of injected MNCs, followed by the lungs and heart. Several cells were also detected in brain tissue.

the transplanted human cells penetrated the blood-brain barrier and entered the damaged brain. Furthermore, the cell number estimates for various organs showed that the liver harvested the highest number of MNCs, followed by the lungs and heart, spleen, kidneys, stomach and brain (Figure 1).

\section{Negative Geotaxis}

In the negative geotaxis test performed on day 7 after cell transplantation (Figure 2), rat pups in the MNC-transplanted group showed a better performance than those in the HIE-only

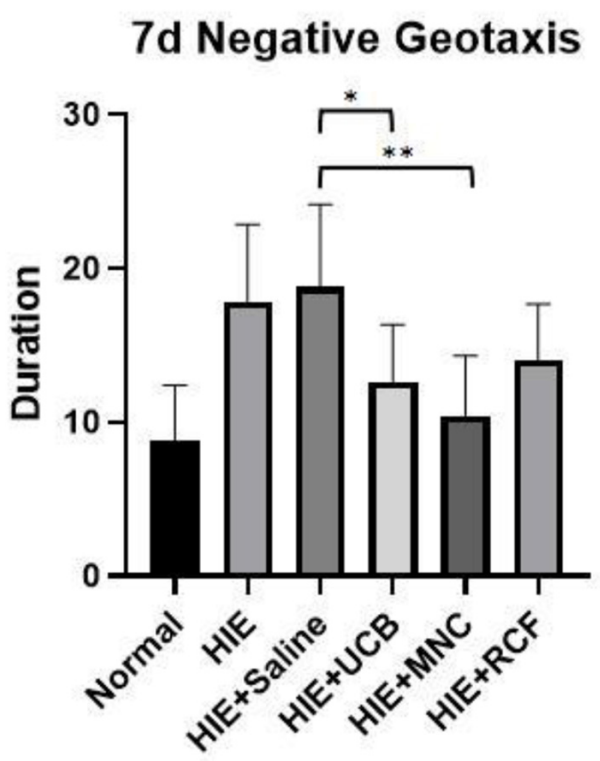

FIGURE 2 | Negative geotaxis results on day 7 after cell transplantation $(n=8)$. The UCB- and MNC-treated groups showed a better performance than the saline-treated HIE and HIE-only groups. "** Indicates $p<0.001$, "**" indicates $p<0.0001$ (one-way ANOVA with Tukey's multiple comparison test).

group ( $p=0.0048)$, but their performance did not reach the level shown by the pups in the normal group. The performances of both the whole blood HUCB-transplanted $(p=0.0088)$ and MNCs-transplanted ( $p=0.0001)$ groups were better than that of the saline-treated HIE group. However, no statistical differences in performance were found between the RCF-treated group and the saline-treated HIE $(p=0.3981)$ and HIE-only $(p=0.7046)$ groups.

\section{Motor Behavior Test}

The beam balance test was performed to assess motor behavior at 1 month (Figure 3A) and 3 months (Figure 3B) after cell transplantation. At 1 month, the UCB-treated $(p=0.0012)$, MNC-treated ( $p=0.0119)$, and RCF-treated ( $p=0.0288)$ groups all scored lower (indicating lesser time) than the HIE-only group, indicating that the three treated groups showed better motor balance and coordination; however, their performance did not reach the level shown by the rat pups in the normal group. Compared with the saline-treated HIE group, both the HUCBtreated $(p=0.0037)$ and MNCs-treated $(p=0.0245)$ groups (but not the RCF-treated group, $p=0.0514$ ) performed better, but their performance did not reach the level shown by the normal group. At 3 months, only the MNC-treated group showed a better motor performance than the HIE-only (0.0022) and salinetreated HIE (0.002) groups, but the performance did not reach the level shown by the normal group.

\section{Morris Water Maze}

The Morris water maze test $(n=12)$ was performed at 1 month (Figure 4A) and 3 months (Figure 4B) after cell transplantation 
A

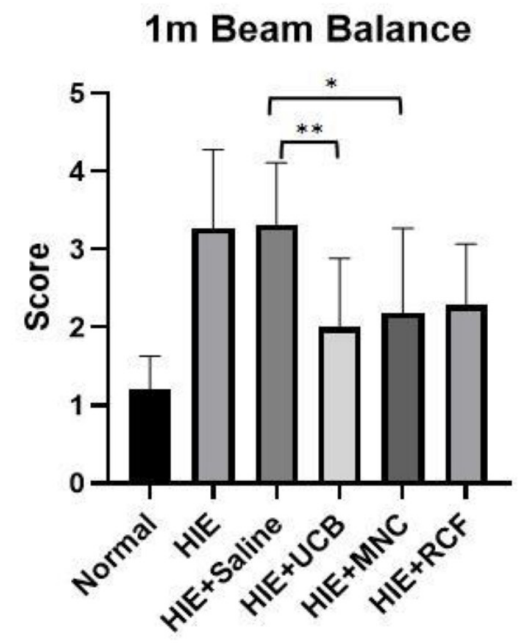

B

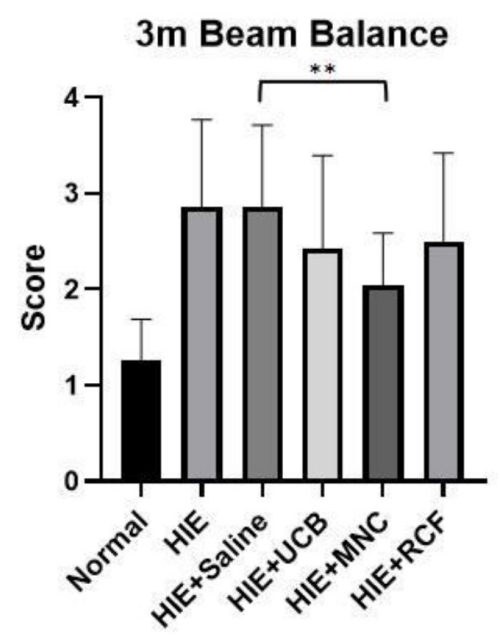

FIGURE 3 | Beam balance results at (A) 1 month and (B) 3 months after cell transplantation $(n=12)$. The MNC-treated group showed better recovery of motor function than the HIE-only and saline-treated groups at both time points ( 1 and 3 months). "** Indicates $p<0.05$, "**" indicates $p<0.005$ (one-way ANOVA with Tukey's multiple comparison test).

to assess the spatial learning memory function of the rat pups. Five-day training trials were conducted at both time points. At 1 month, the performances of the HUCB-treated, MNCs-treated, HIE-only and saline-treated groups on day 3 were significantly different from one another, whereas on day 5 , the rats in both the UCB- and MNC-treated groups found the escape platform sooner than those in the HIE-only and saline-treated groups. At 3 months, no differences were observed in the escape latency between the normal and the three treated groups. In the probe trials at 1 and 3 months, wherein the escape platform was removed, no differences were found between the groups in terms of the cumulative distance and island crossing.

\section{Immunohistochemical Analysis of NeuN-Positive Cells}

At 7 days, 1 and 3 months (Figure 5A) after cell transplantation, paraffin-embedded brain tissue sections were used for an immunohistochemistry study of neurons in the motor-sensory cortex. NeuN-positive cells were counted using software provided by Nikon. The results indicated that on day 7, all HIE groups (both with or without treatments) showed a reduced number of neurons compared with those in normal rats. Although the MNCs-treated group showed a greater number of NeuNpositive cells than the other HIE groups, the differences were not statistically significant (MNCs-treated group vs. HIE-only group, $p=0.818$; vs. saline-treated HIE group, $p=0.1746$ ).

At 1 (Figure 5B) month after cell transplantation, the UCBtreated $(p=0.0024)$, MNC-treated $(p<0.0001)$ and RCFtreated $(p=0.0191)$ groups all showed bigger numbers of neurons compared with the saline-treated HIE group. Compared with the HIE-only group, the HUCB-treated $(p=0.0097)$ and MNCs-treated $(p<0.0001)$ groups had more neurons in the motor-sensory cortex. However, no statistical difference was observed in the number of neurons between the HIE-only and RCF-treated groups $(p=0.0566)$. At 3 months (Figure 5B), both the UCB-treated $(p=0.0154)$ and MNC-treated $(p=0.019)$ groups showed more neurons compared with the saline-treated HIE group. Compared with the HIE-only group, the HUCBtreated $(p=0.0123)$ and MNCs-treated $(p=0.015)$ groups also showed more neurons in the motor-sensory cortex. Even at this time point, however, the number of neurons between the HIEonly and RCF-treated groups $(p=0.0759)$ and between the saline-treated HIE and RCF-treated groups $(p=0.0975)$ were not statistically different. In line with the results of the motor behavior tests, the cell-treated groups had more neurons in the motor-sensory cortex at both 1 - and 3-month time points after cell transplantation, but these numbers did not reach those found in the normal group rats.

\section{DISCUSSION}

In our study, we intravenously transplanted HUCB, MNCs and the RCF into different groups of rat pups $24 \mathrm{~h}$ after HIE modeling. Forty-eight hours after transplantation, human Alu Yb8 expression was detected by real-time PCR to confirm the migration of a small number of the transplanted cells into the brain, contrasting previous studies in the literature (Meier et al., 2006; Yasuhara et al., 2010), where a significant number of human MNCs were present in the grafted brain. To test the short-term effects of cell therapy, the negative geotaxis test was conducted to assess neurological development on day 7 . To test the long-term effects, beam balance and Morris water maze tests were performed to evaluate motor performance and spatial learning respectively, at 1 and 3 months. Furthermore, 
A

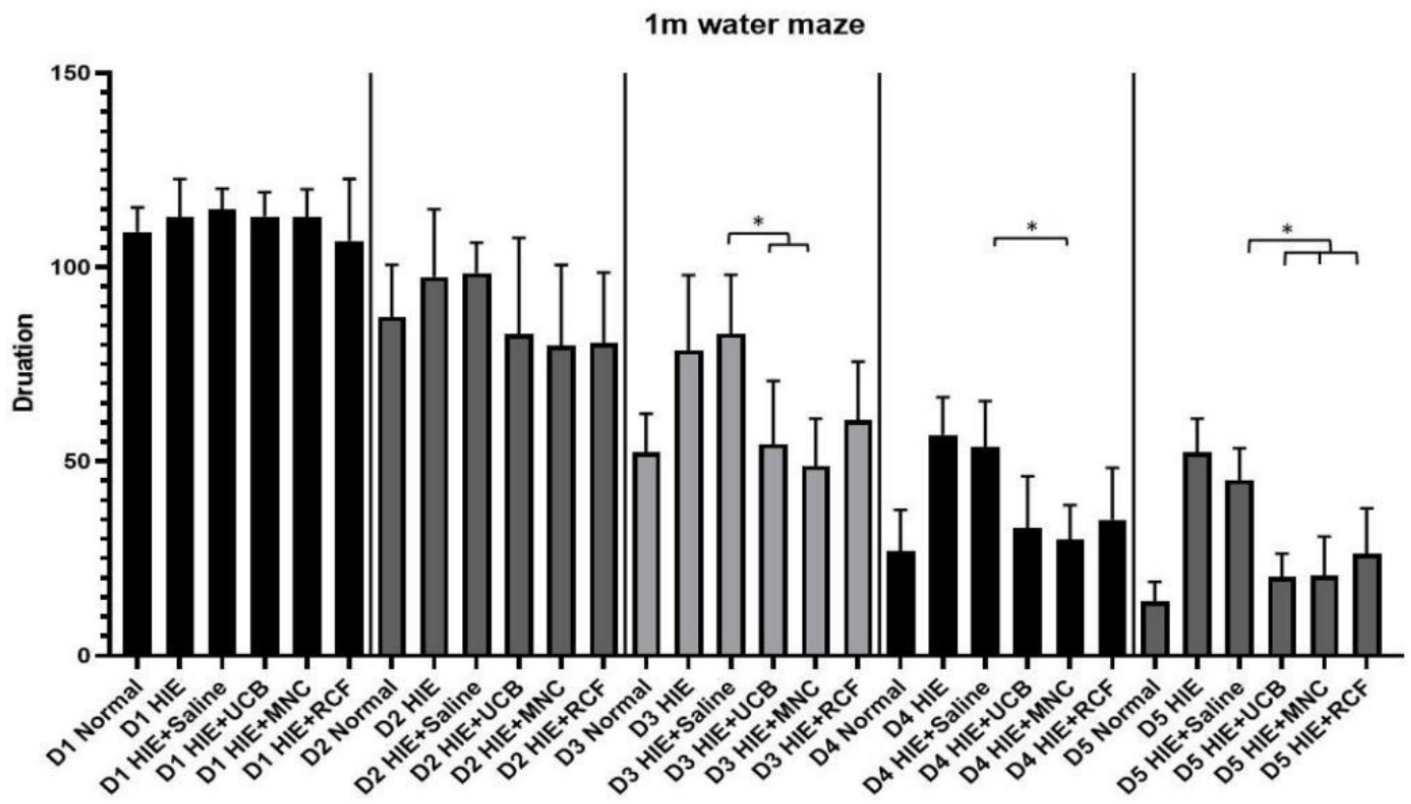

B

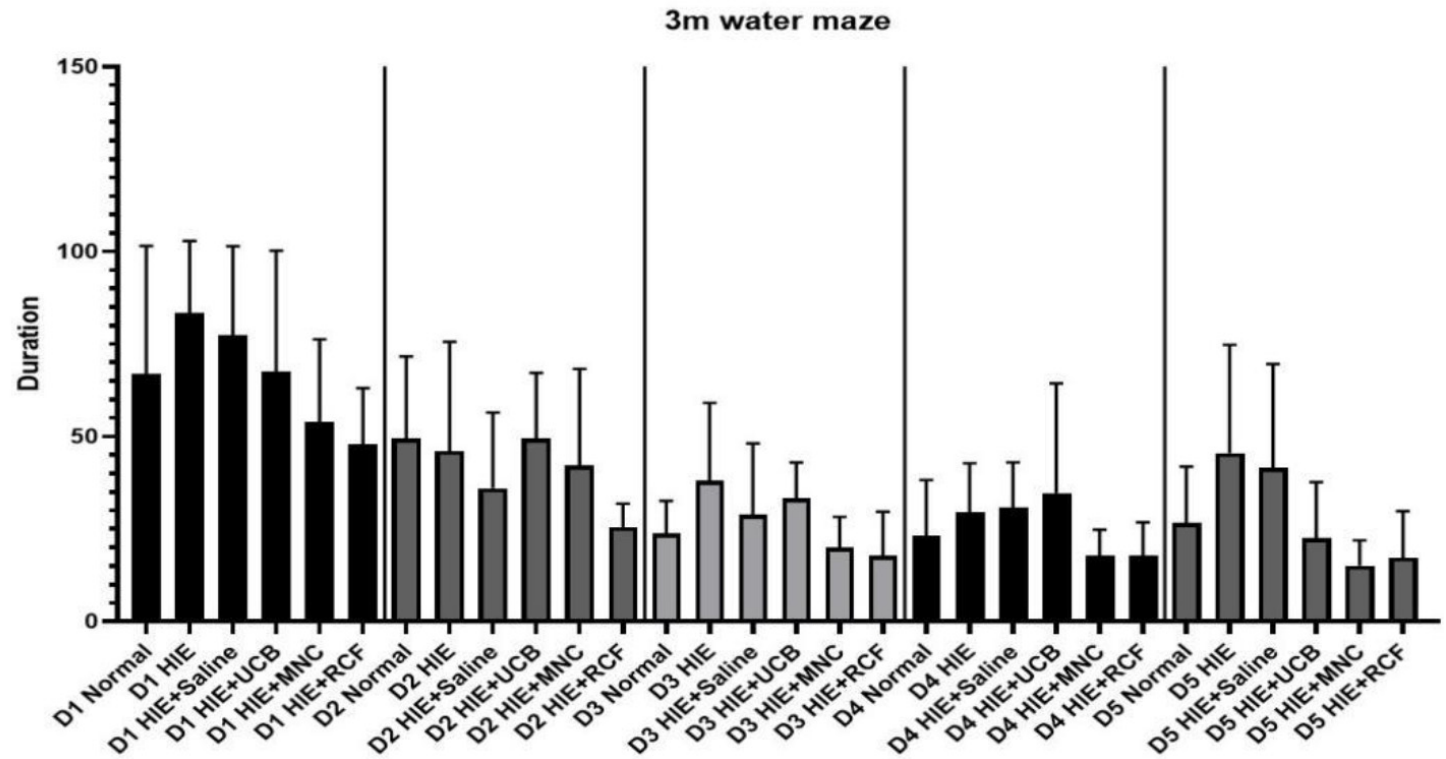

FIGURE 4 | Results of Morris water maze training trials at (A) 1 month and (B) 3 months after cell transplantation $(n=12)$. On day 5 of training at 1 month, the time taken to find the escape platform was shorter in the UCB- and MNC-treated groups than in the saline-treated HIE groups. At 3 months, no difference in the water maze test performance was observed between any group, suggested the spatial learning and memory have not improved further. "** Indicates $p<0.05$ (one-way ANOVA with Tukey's multiple comparison test).

the NeuN immunohistochemistry was carried out to assess the therapeutic efficacy of HUCB and its cellular components (MNCs and RCF) in estimating the number of mature neurons in the motor-sensory cortex.
The day 7 behavioral test demonstrated that MNCs-treated HIE rats performed significantly better in the negative geotaxis suggesting a functional improvement after MNCs-treatment. At 1 month after cell injection, statistically significant differences 
A

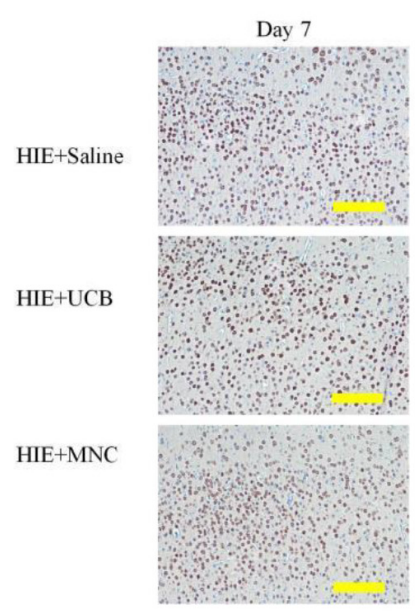

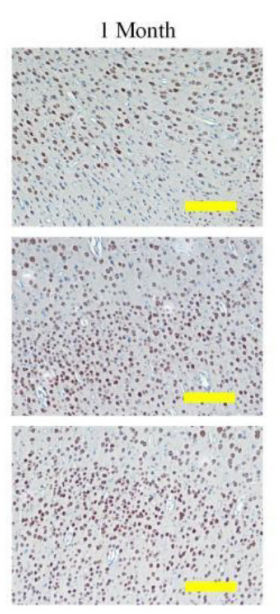

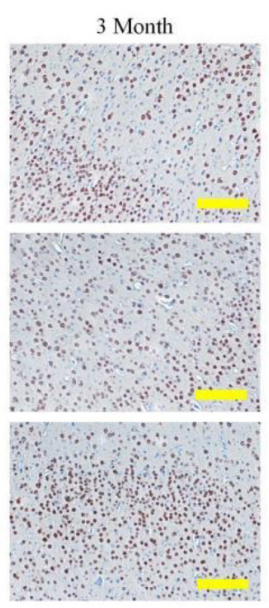

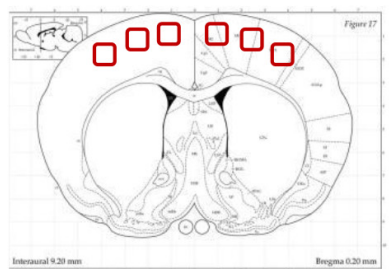

B

i ii

$1 \mathrm{~m}$ Cortex NeuN

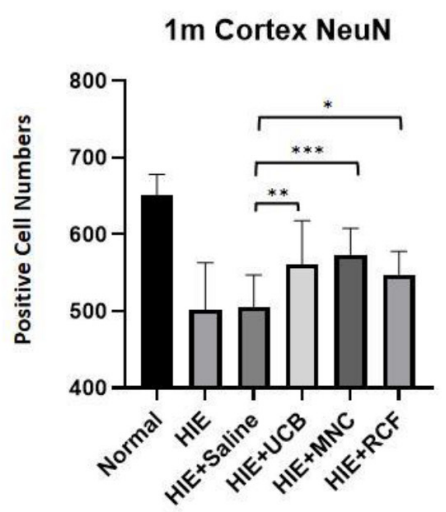

iii

\section{7d Cortex NeuN}

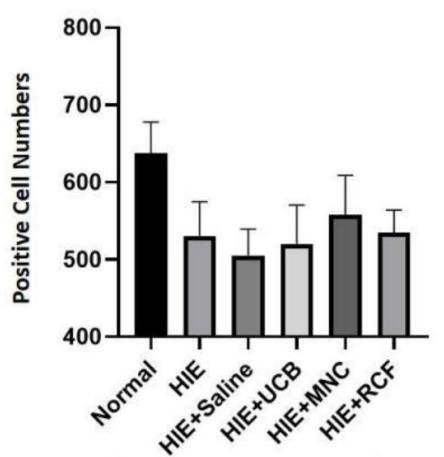

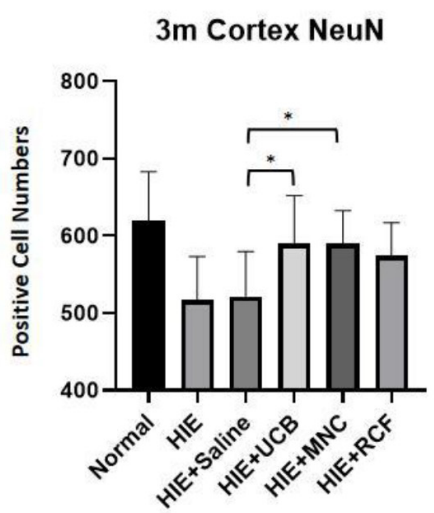

FIGURE 5 | (A) Showed NeuN staining of control, UCB and MNC-treated rats' motor cortex. Scale = $200 \mu \mathrm{m}$. (B) Showed NeuN-positive cell counts at 7 days, 1 and 3 months after cell transplantation $(n=8)$. More NeuN-positive cells were detected in cortex sections from HUCB and MNC-treated rats than in sections from HIE-only and saline-treated HIE rats. "** Indicates $p<0.05$, "*** indicates $p<0.005$, "***" indicates $p<0.0001$ (one-way ANOVA with Tukey's multiple comparison test). Scale $=200 \mu \mathrm{m}$.

were observed between HIE rats and HUCB, MNCs and RCFtreated HIE rats and between saline-treated HIE rats and HUCB and MNCs-treated HIE rats. At 3 months, significant differences were observed between HIE and MNCs-treated HIE rats and between saline-treated HIE rats and MNCs-treated HIE rats. These findings suggest that treatment with HUCB and MNCs led to long-term improvements in the motor function of the rat pups. The favorable treatment outcomes with MNCs are particularly consistent, for the early 7-day, and the long term one and 3 months after the intravenous administration.

The results of histological analysis of NeuN quantification at 1 month after cell injection revealed statistically significant differences between HIE rats and HUCB and MNCs-treated HIE rats and between saline-treated HIE rats and HUCB, MNCs and RCF-treated HIE rats. At 3 months after cell injection, significant differences were found between HIE rats and HUCB and MNCs-treated HIE rats and between saline-treated HIE rats and MNCs-treated HIE rats. These results indicate that MNCs transplantation increases the number of neurons in the motorsensory cortices of HIE rats.

For this current study, there were limitations. For histology, only NeuN immunohistochemistry was employed to quantify the number of mature neurons in the sensory-motor cortex. Other important aspects such as brain water content, brain atrophy and angiogenesis were not investigated. Apoptosis and necrosis were assessed early (Lyu et al., 2021) but not long term. A systematic motor, learning and memory tests were not planned in this study. For future treatment study of HIE, for instance comparing MNCs with exosomes of HUCB, all these and the long-term behavioral tests such as gait 
analysis, forelimb and hind-limb function, and cylinder tests (Balkaya et al., 2018).

In this study, all HUCB and its components were transplanted to HIE rat pups immediately after processing without cryopreservation. The transportation duration was controlled to within one hour and cell viability was maintained over $80 \%$. Weise et al. indicated that cryopreserved HUCB-derived MNCs do not perform well in the treatment of experimental focal cerebral ischemia in achieving favorable long-term neurological recovery, reducing infarct volume and brain atrophy (Weise et al., 2014).

Our findings in HIE rat pups suggest that several potential mechanisms contribute to the therapeutic effects of human HUCB-derived MNCs treatment, namely neuroprotection, neurogenesis and angiogenesis.

\section{Neuroprotection}

On day 7 after MNC transplantation, the MNCs-treated rat pups performed better in the negative geotaxis test compared with HIE control rats. Although quantitative analyses of NeuN expression in the brain cortex showed no statistical difference between the MNC-treated and HIE groups, the average number of neurons was higher in the MNCs-treated rat cortex. These results suggest a potential neuroprotective effect against HIE within 1 week after MNC transplantation. A previous study reported a similar neuroprotective effect of MNCs in an in vitro cell culture experiment and suggested that multiple MNC types contribute to this effect (Reich et al., 2008).

\section{Neurogenesis}

The quantitative analysis of NeuN expression demonstrated that the average number of cortical neurons in the HIE rat pups increased at 1 and 3 months after cell transplantation, compared with that on day 7 after cell transplantation. This suggests a potential neurogenic effect on the damaged brain areas of HIE rats. In line with this result, freshly isolated HUCB-derived MNCs have been reported to express brain-derived neurotrophic factor (BDNF), which plays a role in reducing neuronal cell death and stimulating neurogenesis, thereby enhancing the regeneration of damaged brain areas after injury (Bath and Lee, 2010; Nagahara and Tuszynski, 2011).

\section{Angiogenesis}

One recent study has demonstrated that bone marrow derived MNCs suppressed autophagy in focal ischemia mice by activating angiogenesis through gap junction-mediated signaling. They also proved this procedure was induced by activation of hypoxia inducible factor (HIF)-1 $\alpha$ (Kikuchi-Taura et al., 2020). As bone marrow MNCs shows a similar cell component, angiogenesis could act as another potential mechanism of HUCB-MNCs transplantation.

They further indicated that bone marrow MNCs restored cerebral metabolism after their transplantation to aging mice resulting in improve neurological functions. The underlying mechanism is related to glucose transporter expression level and activity of $\mathrm{Na}+/ \mathrm{K}+$-ATPase in mice brain (Takeuchi et al., 2020).
Hypoxic-ischemic encephalopathy is a serious condition in new-born babies that may result in permanent motor dysfunction, such as $\mathrm{CP}$, or even death. In recent decades, hypothermia therapy has been established to be an effective intensive care treatment modality for neonates with HIE. However, this therapy has not been able to decrease the mortality rate among HIE cases to below the 20\% (Shankaran et al., 2005). Therefore, additional novel therapy development, both in mortality and disability reduction, is urgently required. Currently, many preclinical studies are investigating the efficacy of HUCB and bone marrow-derived stem cells (Nabetani et al., 2018).

In this 40-year period, a number of animal studies have suggested a few mechanisms where HUCB may exert its therapeutic effects against HIE. Five stages of the HIE processes have been identified: (1) energy consumption, (2) inflammation, (3) cellular excitotoxicity, (4) oxidative stress and (5) cell death. Specifically, HUCB treatment has been hypothesized to ameliorate inflammation and oxidative stress, preventing apoptosis and promoting brain regeneration (Epstein and McCord, 1985; Ferriero, 2004; Johnston et al., 2011).

It remains unclear which specific components of the HUCB is the major contributor to its anti-inflammatory effect in HIE treatment. Studies have shown that HUCB-derived MSCs and endothelial progenitor cells exert inflammation-ameliorating effects after brain injury. In addition, MSCs can regulate immune reactions, which may be beneficial against both local and global neuro-inflammatory processes occurring in HIE (Wasielewski et al., 2012; Rosenkranz et al., 2013; Paton et al., 2017). Some studies have demonstrated that intraperitoneal injection of HUCB-derived MNCs $6 \mathrm{~h}$ after ischemia could decrease cell death and free radicals (Rosenkranz et al., 2012; Hattori et al., 2015). Another type of human-derived cells, such as CD34 ${ }^{+}$ hematopoietic stem cells, have been shown to secrete numerous growth factors, including BDNF, glial cell-derived neurotrophic factor and vascular endothelial growth factor (VEGF), all of which could promote recovery from brain damage in HIE (Yoshihara et al., 2008; Rosenkranz et al., 2010). CD34 ${ }^{+}$cells have also been reported to provide an environment beneficial for neuronal regeneration after ischemic stroke (Taguchi et al., 2004a,b). In our study, based on real-time PCR results, organs like liver, lung and heart harvested large amounts of injected cells, these cells may also have the possibility of releasing cytokines, growth factors, neurotrophic factors or other small molecules. These factors may enter the brain through an impaired bloodbrain barrier (BBB).

Human umbilical cord blood is a well-known source of a variety of stem cells, including $\mathrm{CD} 34^{+}$cells and endothelial progenitor cells. The proportion of $\mathrm{CD}_{3} 4^{+}$cells in HUCB is approximately $1-2 \%$, in comparison with less than $0.01 \%$ in adult human peripheral blood (Murohara et al., 2000; Rafii and Lyden, 2003; Sun et al., 2010). HUCB treatments have been shown promising effects against hematological diseases, including leukemia, and this treatment method has replaced the use of hematopoietic stem cells in the last few decades (Tezuka et al., 2002; Gluckman and Rocha, 2004). Furthermore, as a source of endothelial stem cells, HUCB has shown 
therapeutic effects against many diseases, such as CP, diabetes and cardiovascular diseases (Lindvall and Kokaia, 2006).

Many studies have focused on the use of MNCs to treat HIE. Sharma et al. (2015) reported that bone marrowderived MNC therapy led to behavioral improvements in patients with $\mathrm{CP}$ at the 6-month follow-up. Nuclear Medicine Imaging showed improved metabolism in damaged brain areas in these patients, which were consistent with the observed clinical improvements. Based on these results, the researchers concluded that MNC therapy can stimulate neurological development, reduce brain dysfunction and promote the quality of life in patients with $\mathrm{CP}$ (Sharma et al., 2015).

Translating bench research findings into clinical trials in a timely and methodologically appropriate manner, it is logical to follow the adult stroke community's STEP 4 movement, using stem cells therapy as an emerging paradigm to advance and to accelerate preclinical research (Boltze et al., 2019) as HIE is a form of neonatal ischemia. A recent example of the timing of specific and intensive stroke rehabilitation directly translated from rodent experiments to human, showing rehabilitation early within 30 days and late beyond 6 months are not effective. The most effective period has been proven to be 60-90 days (Dromerick et al., 2021). Our experimental work on HUCB and its MNCs treating HIE can be fast-tracked to clinical trials when its availability, dosage and timing are worked out.

\section{CONCLUSION}

Our data suggest that

(1) HUCB transplantation can improve short-term and partially long-term neurological function in neonatal rats with HIE.

(2) RCF has a partial long-term treatment effect in neonatal rats with HIE.

(3) HUCB-derived MNC is an effective therapy that can improve both short-term and long-term neurological function in neonatal rats with HIE.

\section{REFERENCES}

Azzopardi, D. V., Strohm, B., Edwards, A. D., Dyet, L., Halliday, H. L., Juszczak, E., et al. (2009). Moderate Hypothermia to Treat Perinatal Asphyxial Encephalopathy. N. Engl. J. Med. 361, 1349-1358. doi: 10.1056/nejmoa0900854

Balkaya, M. G., Trueman, R. C., Boltze, J., Corbett, D., and Jolkkonen, J. (2018). Behavioral Outcome Measures to Improve Experimental Stroke Research. Behav. Brain Res. 352, 161-171. doi: 10.1016/J.BBR.2017.07.039

Bath, K. G., and Lee, F. S. (2010). Neurotrophic Factor Control of Adult SVZ Neurogenesis. Dev. Neurobiol. 70, 339-349. doi: 10.1002/dneu.20781

Bennet, L., Tan, S., Van Den Heuij, L., Derrick, M., Groenendaal, F., Van Bel, F., et al. (2012). Cell Therapy for Neonatal Hypoxia-Ischemia and Cerebral Palsy. Ann. Neurol. 71, 589-600. doi: 10.1002/ana.22670

Boltze, J., Modo, M. M., Mays, R. W., Taguchi, A., Jolkkonen, J., and Savitz, S. I. (2019). Stem Cells as an Emerging Paradigm in Stroke 4 Advancing and Accelerating Preclinical Research. Stroke 50, 3299-3306. doi: 10.1161/ STROKEAHA.119.025436

\section{DATA AVAILABILITY STATEMENT}

The original contributions presented in the study are included in the article/supplementary material, further inquiries can be directed to the corresponding author/s.

\section{ETHICS STATEMENT}

The animal study was reviewed and approved by the Ethics Committee of The Chinese University of Hong Kong.

\section{AUTHOR CONTRIBUTIONS}

WP, WY, and CW conceived and designed the study. HL contributed to the animal experiments, behavioral tests, histology and statistical analysis. JC and YH contributed to the animal observation and care. SL and $\mathrm{ZZ}$ contributed to the cell quantification. DS, $\mathrm{CN}$, and $\mathrm{GH}$ contributed to the technical support. WP, WY, CW, DS, and CN supervised the study. $\mathrm{HL}$ drafted the manuscript. WP, $\mathrm{CN}$, and $\mathrm{WY}$ revised the manuscript. All authors contributed to the article and approved the submitted version.

\section{FUNDING}

This study was supported by the Innovation and Technology Fund (ITS-208-16FX) from Innovation and Technology Commission, Theme-based Research Scheme (T13-602/21-N), and Collaborative Research Fund (C5045-20EF) from Research Grant Council, Hong Kong.

\section{ACKNOWLEDGMENTS}

We like to express our gratitude to G. Martino from Universita Vita-Salute, San Raffaele, who has critically appraised $\mathrm{H}$. Lyu's Ph.D. thesis, which contains the core materials of this manuscript, and Wendy Cheng of Mononuclear Therapeutics Ltd for providing the HUCB.

Cotten, C. M., Murtha, A. P., Goldberg, R. N., Grotegut, C. A., Smith, P. B., Goldstein, R. F., et al. (2014). Feasibility of Autologous Cord Blood Cells for Infants with Hypoxic-Ischemic Encephalopathy. J. Pediatr. 164, 973-979.e1. doi: 10.1016/j.jpeds.2013.11.036

Douglas-Escobar, M., and Weiss, M. D. (2015). Hypoxic-Ischemic Encephalopathy A Review for the Clinician. JAMA Pediatr. 169, 397-403. doi: 10.1001/ jamapediatrics.2014.3269

Dromerick, A. W., Geed, S., Barth, J., Brady, K., Giannetti, M. L., Mitchell, A., et al. (2021). Critical Period After Stroke Study (CPASS): a phase II clinical trial testing an optimal time for motor recovery after stroke in humans. Proc. Natl. Acad. Sci. U. S. A. 118:E2026676118. doi: 10.1073/pnas.20266 76118

Eklind, S., Mallard, C., Arvidsson, P., and Hagberg, H. (2005). Lipopolysaccharide Induces Both a Primary and a Secondary Phase of Sensitization in the Developing Rat Brain. Pediatr. Res. 58, 112-116. doi: 10.1203/01.PDR.0000163513.036 $19.8 \mathrm{D}$ 
Epstein, F. H., and McCord, J. M. (1985). Oxygen-Derived Free Radicals in Postischemic Tissue Injury. N. Engl. J. Med. 312, 159-163. doi: 10.1056/ nejm 198501173120305

Ferriero, D. M. (2004). Neonatal Brain Injury. N. Engl. J. Med. 351, 1985-1995. doi: $10.1056 /$ nejmra041996

Gluckman, E., and Rocha, V. (2004). Cord Blood Transplantation for Children with Acute Leukaemia: a Eurocord Registry Analysis. Blood Cells Mol. Dis. 33, 271-273. doi: 10.1016/j.bcmd.2004.08.015

Gluckman, P. D., Wyatt, J. S., Azzopardi, D., Ballard, R., Edwards, A. D., and Ferriero, D. M. (2005). Neonatal Encephalopathy: multicentre Randomised Trial. Lancet 365, 663-670. doi: 10.1016/S0140-6736(05)17946-X

Graham, H. K., Rosenbaum, P., Paneth, N., Dan, B., Lin, J. P., Damiano, J. G., et al. (2016). Cerebral Palsy. Nat. Rev. Dis. Primers 2:15082. doi: 10.1038/nrdp. 2015.82

Gunn, A. J., Gunn, T. R., De Haan, H. H., Williams, C. E., and Gluckman, P. D (1997). Dramatic Neuronal Rescue with Prolonged Selective Head Cooling after Ischemia in Fetal Lambs. J. Clin. Invest. 99, 248-256. doi: 10.1172/JCI119153

Gunn, A. J., Wyatt, J. S., Whitelaw, A., Barks, J., Azzopardi, D., Ballard, R., et al. (2008). Therapeutic Hypothermia Changes the Prognostic Value of Clinical Evaluation of Neonatal Encephalopathy. J. Pediatr. 152, 55-58. doi: 10.1016/j. jpeds.2007.06.003

Hattori, T., Sato, Y., Kondo, T., Ichinohashi, Y., Sugiyama, Y., Yamamoto, M., et al. (2015). Administration of Umbilical Cord Blood Cells Transiently Decreased Hypoxic-Ischemic Brain Injury in Neonatal Rats. Dev. Neurosci. 37, 95-104. doi: $10.1159 / 000368396$

Higgins, R. D., Raju, T., Edwards, A. D., Azzopardi, A. D., Bose, C. L., Clark, R. H., et al. (2011). Hypothermia and Other Treatment Options for Neonatal Encephalopathy: an Executive Summary of the Eunice Kennedy Shriver NICHD Workshop. J. Pediatr. 159, 851-858e1. doi: 10.1016/j.jpeds2011. 08.004

Jacobs, S. E., Morley, C. J., Inder, T. E., Stewart, M. J., Smith, K. R., McNamara, P. J., et al. (2011). Whole-Body Hypothermia for Term and near-Term Newborns with Hypoxic-Ischemic Encephalopathy: a Randomized Controlled Trial. Arch. Pediatr. Adolesc. Med. 165, 692-700. doi: 10.1001/archpediatrics.2011.43

Jaing, T.-H. (2014). Umbilical Cord Blood: a Trustworthy Source of Multipotent Stem Cells for Regenerative Medicine. Cell Transplant. 23, 493-496. doi: 10. 3727/096368914X678300

Jantzie, L. L., Scafidi, J., and Robinson, S. (2018). Stem Cells and Cell-Based Therapies for Cerebral Palsy: a Call for Rigor. Pediatr. Res. 83, 345-355. doi: 10.1038/pr.2017.233

Johnston, M. V., Fatemi, A., Wilson, M. A., and Northington, F. (2011). Treatment Advances in Neonatal Neuroprotection and Neurointensive Care. Lancet Neurol. 10, 372-382. doi: 10.1016/S1474-4422(11)70016-3

Kikuchi-Taura, A., Okinaka, Y., Takeuchi, Y., Ogawa, Y., Maeda, M., Kataoka, Y., et al. (2020). Bone Marrow Mononuclear Cells Activate Angiogenesis via Gap Junction-Mediated Cell-Cell Interaction. Stroke 51, 1279-1289. doi: 10.1161/ STROKEAHA.119.028072

Kurinczuk, J. J., White-Koning, M., and Badawi, N. (2010). Epidemiology of Neonatal Encephalopathy and Hypoxic-Ischaemic Encephalopathy. Early Hum. Dev. 86, 329-338. doi: 10.1016/j.earlhumdev.2010.05.010

Lehnardt, S., Massillon, L., Follett, P., Jensen, F. E., Ratan, R., Rosenberg, P. A., et al. (2003). Activation of Innate Immunity in the CNS Triggers Neurodegeneration through a Toll-like Receptor 4-Dependent Pathway. Proc. Natl. Acad. Sci. U. S. A. 100, 8514-8519. doi: 10.1073/pnas. 1432609100

Lindvall, O., and Kokaia, Z. (2006). Stem Cells for the Treatment of Neurological Disorders. Nature 441, 1094-1096. doi: 10.1038/nature04960

Lyu, H., Sun, D. M., Ng, C. P., Chen, J. F., He, Y. Z., Lam, S. Y., et al. (2021). A New Hypoxic Ischemic Encephalopathy Model in Neonatal Rats. Heliyon 7:e08646. doi: 10.1016/J.HELIYON.2021.E08646

Meier, C., Middelanis, J., Wasielewski, B., Neuhoff, S., Roth-Haerer, A., Gantert, M., et al. (2006). Spastic Paresis after Perinatal Brain Damage in Rats Is Reduced by Human Cord Blood Mononuclear Cells. Pediatr. Res. 59, 244-249. doi: 10.1203/01.pdr.0000197309.08852.f5

Murohara, T., Ikeda, H., Duan, J., Shintani, S., Sasaki, K. I., Eguchi, H., et al. (2000). Transplanted Cord Blood-Derived Endothelial Precursor Cells Augment Postnatal Neovascularization. J. Clin. Invest. 105, 1527-1536. doi: 10.1172/JCI8296
Nabetani, M., Shintaku, H., and Hamazaki, T. (2018). Future Perspectives of Cell Therapy for Neonatal Hypoxic-Ischemic Encephalopathy. Pediatr. Res. 83, 356-363. doi: 10.1038/pr.2017.260

Nagahara, A. H., and Tuszynski, M. H. (2011). Potential Therapeutic Uses of BDNF in Neurological and Psychiatric Disorders. Nat. Rev. Drug Discov. 10, 209-219. doi: $10.1038 / \mathrm{nrd} 3366$

Paton, M. C. B., McDonald, C. A., Allison, B. J., Fahey, M. C., Jenkin, G., and Miller, S. L. (2017). Perinatal Brain Injury as a Consequence of Preterm Birth and Intrauterine Inflammation: designing Targeted Stem Cell Therapies. Front. Neurosci. 11:200. doi: 10.3389/fnins.2017.00200

Penny, T. R., Sutherland, A. E., Mihelakis, J. G., Paton, M. C. B., Pham, Y., and Lee, J. (2019). Human Umbilical Cord Therapy Improves Long-Term Behavioral Outcomes Following Neonatal Hypoxic Ischemic Brain Injury. Front. Physiol. 10:283. doi: 10.3389/FPHYS.2019.00283

Pimentel-Coelho, P. M., and Mendez-Otero, R. (2010). Cell Therapy for Neonatal Hypoxic-Ischemic Encephalopathy. Stem Cells Dev. 19, 299-309.

Rafii, S., and Lyden, D. (2003). Therapeutic Stem and Progenitor Cell Transplantation for Organ Vascularization and Regeneration. Nat. Med. 9, 702-712. doi: 10.1038/nm0603-702

Reich, D. M., Hau, S., Stahl, T., Scholz, M., Naumann, W., Emmrich, F., et al. (2008). Neuronal Hypoxia in Vitro: investigation of Therapeutic Principles of HUCB-MNC and CD133+ Stem Cells. BMC Neurosci. 9:91. doi: 10.1186/14712202-9-91

Rice, J. E., Vannucci, R. C., and Brierley, J. B. (1981). The Influence of Immaturity on Hypoxic-ischemic Brain Damage in the Rat. Ann. Neurol. 9, 131-141. doi: 10.1002/ana.410090206

Rosenkranz, K., Kumbruch, S., Lebermann, K., Marschner, K., Jensen, A., Dermietzel, R., et al. (2010). The Chemokine SDF-1/CXCL12 Contributes to the 'homing' of Umbilical Cord Blood Cells to a Hypoxic-Ischemic Lesion in the Rat Brain. J. Neurosci. Res. 88, 1223-1233. doi: 10.1002/jnr. 22292

Rosenkranz, K., Kumbruch, S., Tenbusch, M., Marcus, K., Marschner, K., Dermietzel, R., et al. (2012). Transplantation of Human Umbilical Cord Blood Cells Mediated Beneficial Effects on Apoptosis, Angiogenesis and Neuronal Survival after Hypoxic-Ischemic Brain Injury in Rats. Cell Tissue Res. 348, 429-438. doi: 10.1007/s00441-012-1401-0

Rosenkranz, K., Tenbusch, M., May, C., Marcus, K., and Meier, C. (2013). Changes in Interleukin-1 Alpha Serum Levels after Transplantation of Umbilical Cord Blood Cells in a Model of Perinatal Hypoxic-Ischemic Brain Damage. Ann. Anat. 195, 122-127. doi: 10.1016/j.aanat.2012.09.003

Shankaran, S., Laptook, A. R., Ehrenkranz, R. A., Tyson, J. E., McDonald, S. A., Donovan, E. F., et al. (2005). Whole-Body Hypothermia for Neonates with Hypoxic-Ischemic Encephalopathy. N. Engl. J. Med. 353, 1574-1584. doi: 10. 1056/nejmcps050929

Sharma, A., Sane, H., Gokulchandran, N., Kulkarni, P., Gandhi, S., Sundaram, J., et al. (2015). A clinical study of autologous bone marrow mononuclear cells for cerebral palsy patients: a new frontier. Stem Cells Int. 2015:905874. doi: $10.1155 / 2015 / 905874$

Sun, J., Allison, J., McLaughlin, C., Sledge, L., Waters-Pick, B., Wease, S., et al. (2010). Differences in quality between privately and publicly banked umbilical cord blood units: a pilot study of autologous cord blood infusion in children with acquired neurologic disorders. Transfusion 50, 1980-1987. doi: 10.1111/j. 1537-2995.2010.02720.x

Taguchi, A., Matsuyama, T., Moriwaki, H., Hayashi, T., Hayashida, K., Nagatsuka, K., et al. (2004a). Circulating CD34-Positive Cells Provide an Index of Cerebrovascular Function. Circulation 109, 2972-2975. doi: 10.1161/01.CIR. 0000133311.25587.DE

Taguchi, A., Soma, T., Tanaka, H., Kanda, T., Nishimura, H., Yoshikawa, H., et al. (2004b). Administration of CD34+ Cells after Stroke Enhances Neurogenesis via Angiogenesisin a Mouse Model. J. Clin. Invest. 114, 330-338. doi: 10.1172/ jci20622

Takeuchi, Y., Okinaka, Y., Ogawa, Y., Kikuchi-Taura, A., Kataoka, Y., Gul, S., et al. (2020). Intravenous Bone Marrow Mononuclear Cells Transplantation in Aged Mice Increases Transcription of Glucose Transporter 1 and $\mathrm{Na}+/ \mathrm{K}+$-ATPase at Hippocampus Followed by Restored Neurological Functions. Front. Aging Neurosci. 12:170. doi: 10.3389/FNAGI.2020.00170 
Tezuka, K., Nakayama, H., Honda, K., Suzumiya, J., Oshima, K., Kitoh, T., et al. (2002). Treatment of a Child with Myeloid/NK Cell Precursor Acute Leukemia with L-Asparaginase and Unrelated Cord Blood Transplantation. Int. J. Hematol. 75, 201-206. doi: 10.1007/BF02982029

Vorhees, C. V., and Williams, M. T. (2006). Morris water maze: procedures for assessing spatial and related forms of learning and memory. Nat. Protoc. 1, 848-858. doi: 10.1038/nprot.2006.116

Wasielewski, B., Jensen, A., Roth-Härer, A., Dermietzel, R., and Meier, C. (2012). Neuroglial Activation and $\mathrm{Cx} 43$ Expression Are Reduced upon Transplantation of Human Umbilical Cord Blood Cells after Perinatal Hypoxic-Ischemic Injury. Brain Res. 1487, 39-53. doi: 10.1016/j.brainres.2012. 05.066

Weise, G., Lorenz, M., Pösel, C., Riegelsberger, U. M., Störbeck, V., Kamprad, M., et al. (2014). Transplantation of Cryopreserved Human Umbilical Cord Blood Mononuclear Cells Does Not Induce Sustained Recovery after Experimental Stroke in Spontaneously Hypertensive Rats. J. Cereb. Blood Flow Metab. 34, 182-184. doi: 10.1038/JCBFM.2013.185

Yasuhara, T., Hara, K., Maki, M., Xu, L., Yu, G., Ali, M. M., et al. (2010). Mannitol Facilitates Neurotrophic Factor Up-Regulation and Behavioural Recovery in Neonatal Hypoxic-Ischaemic Rats with Human Umbilical Cord Blood Grafts. J. Cell Mol. Med. 14, 914-921. doi: 10.1111/j.1582-4934.2008. 00671.x

Yoshihara, T., Taguchi, A., Matsuyama, T., Shimizu, Y., Kikuchi-Taura, A., Soma, T., et al. (2008). Increase in Circulating CD34-Positive Cells in Patients with Angiographic Evidence of Moyamoya-like Vessels. J. Cereb. Blood Flow Metab. 28, 1086-1089. doi: $10.1038 / j \mathrm{cbfm} .2008 .1$
Zhu, H., Poon, W., Liu, Y., Leung, G. K., Wong, Y., Feng, Y., et al. (2016). Phase I-II Clinical Trial Assessing Safety and Efficacy of Umbilical Cord Blood Mononuclear Cell Transplant Therapy of Chronic Complete Spinal Cord Injury. Cell Transplant. 25, 1925-1943. doi: 10.3727/096368916X6 91411

Conflict of Interest: WC was employed by the company Mononuclear Therapeutics Limited.

The remaining authors declare that the research was conducted in the absence of any commercial or financial relationships that could be construed as a potential conflict of interest.

Publisher's Note: All claims expressed in this article are solely those of the authors and do not necessarily represent those of their affiliated organizations, or those of the publisher, the editors and the reviewers. Any product that may be evaluated in this article, or claim that may be made by its manufacturer, is not guaranteed or endorsed by the publisher.

Copyright $\odot 2022$ Lyu, Sun, Ng, Cheng, Chen, He, Lam, Zheng, Huang, Wang, Young and Poon. This is an open-access article distributed under the terms of the Creative Commons Attribution License (CC BY). The use, distribution or reproduction in other forums is permitted, provided the original author(s) and the copyright owner(s) are credited and that the original publication in this journal is cited, in accordance with accepted academic practice. No use, distribution or reproduction is permitted which does not comply with these terms. 